\title{
Closed surface bundles of least volume
}

\author{
JOHN W AABER \\ NATHAN DUNFIELD
}

\begin{abstract}
Since the set of volumes of hyperbolic 3-manifolds is well ordered, for each fixed $g$ there is a genus $-g$ surface bundle over the circle of minimal volume. Here, we introduce an explicit family of genus $-g$ bundles which we conjecture are the unique such manifolds of minimal volume. Conditional on a very plausible assumption, we prove that this is indeed the case when $g$ is large. The proof combines a soft geometric limit argument with a detailed Neumann-Zagier asymptotic formula for the volumes of Dehn fillings.

Our examples are all Dehn fillings on the sibling of the Whitehead manifold, and we also analyze the dilatations of all closed surface bundles obtained in this way, identifying those with minimal dilatation. This gives new families of pseudo-Anosovs with low dilatation, including a genus 7 example which minimizes dilatation among all those with orientable invariant foliations.
\end{abstract}

57M50; 37E30, 37E40

\section{Introduction}

\subsection{Volumes of bundles}

Thurston and Jørgensen proved that the set of volumes of hyperbolic 3-manifolds is a well-ordered set of type $\omega^{\omega}$. In particular, any topological class of hyperbolic 3-manifolds contains one of least volume. Recent work of Gabai, Meyerhoff and Milley has provably identified the hyperbolic manifold of least volume, and, for instance, the smallest orientable manifolds with one and two cusps are also known (see their recent survey [7] for more).

Here, we focus on closed hyperbolic 3-manifolds which are surface bundles over the circle. Fix a genus $g$, and let $\Sigma_{g}$ denote the closed surface of that genus. Consider all hyperbolic 3-manifolds which are $\Sigma_{g}$-bundles over the circle. As discussed, there is at least one such manifold of least volume, and one goal here is to identify these manifolds. 
To this end, in Section 3 we define for each $g>2$ a certain $\Sigma_{g}$-bundle $M_{g}$ as an explicit Dehn filling on the Whitehead sibling manifold $W$. For instance, if $g \equiv 0,1 \bmod 5$, then $M_{g}=W(g /(2 g+5),-(2 g+3) /(g+4))$. We conjecture that the $M_{g}$ are the minimal volume genus $-g$ bundles for all $g>2$. For large $g$, we prove this contingent on a very plausible conjecture about closed hyperbolic 3-manifolds with $b_{1}(N)=\operatorname{dim} H_{1}(N ; \mathbb{Q}) \geq 2$. In particular, we show:

1.2 Theorem Assume that any closed hyperbolic 3-manifold $N$ with $b_{1}(N) \geq 2$ has volume at least $V_{8} \approx 3.663862$. Then for all large $g$, the manifold $M_{g}$ is the unique $\Sigma_{g}$-bundle of minimal volume.

We give evidence for the needed assumption in Section 4. Alternatively, our proof of Theorem 1.2 shows:

\subsection{Theorem Either}

(1) $M_{g}$ is the unique $\Sigma_{g}$-bundle of minimal volume for all large $g$, or

(2) there is a single closed hyperbolic 3-manifold $N$ which is the minimal volume $\Sigma_{g}$-bundle for infinitely many $g$. (In particular, $b_{1}(N) \geq 2$.)

The proof of Theorem 1.2 has two main parts. The first, Theorem 4.3, is the qualitative statement that the minimal volume $\Sigma_{g}$-bundles, for large $g$, must be Dehn fillings on the Whitehead sibling $W$. This is proved by combining a soft geometric limit argument with work of Agol [1] on volumes of cusped manifolds. Then, in Theorem 5.1, we sift through the large number of $\Sigma_{g}$-bundles that arise from $W$ and find the one with least volume. To do this, in Section 5 we work out the first difficult term of the Neumann-Zagier asymptotic expansion for volumes of Dehn fillings of $W$.

\subsection{Dilatations of pseudo-Anosovs}

We now discuss another minimization problem that turns out to be closely related to finding the smallest volume surface bundles. A pseudo-Anosov homeomorphism $\psi$ of $\Sigma_{g}$ has an associated dilatation $\lambda>1$, also called the stretch-factor or growth, which measures how the invariant foliations are distorted. The number $\lambda$ is an algebraic integer, and $\log (\lambda)$ is the topological entropy of the map $\psi$. For each fixed genus, there is a smallest such dilatation, which we denote $\delta_{g}$. There has been much interest in determining $\delta_{g}$, in part because $\log \delta_{g}$ is the length of the shortest geodesic in the Teichmüller metric on the moduli space of curves.

For a pseudo-Anosov $\psi$ of $\Sigma_{g}$, its mapping torus $M_{\psi}$ is a hyperbolic 3-manifold. Conversely, a hyperbolic 3-manifold which is a $\Sigma_{g}$-bundle over the circle must have 
pseudo-Anosov monodromy. It is natural to ask whether there is a connection between the dilatation of $\psi$ and the volume of $M_{\psi}$. Recently, Farb, Leininger and Margalit have shown that, qualitatively, pseudo-Anosovs with low dilatations come from manifolds with low volume.

1.5 Theorem [5] For each $L>0$, there exist finitely many finite-volume hyperbolic 3-manifolds $N_{1}, \ldots, N_{n}$ so that the following holds. If $\psi$ is a pseudo-Anosov homeomorphism of a surface $\Sigma$ with $\chi(\Sigma)<0$ and $\log (\lambda(\psi))<(1 /|\chi(\Sigma)|) L$, the bundle $M_{\psi}$ is a Dehn filling on one of the $N_{k}$. In particular, there is a $V=V(L)$ so that $\operatorname{Vol}\left(M_{\psi}\right) \leq V$.

Moreover, they show that the fibration of $M_{\psi}$ comes from a fibration of $N_{k}$ that extends over the solid tori added by Dehn filling. Thus, in particular, the bundles coming from the minimal dilatation pseudo-Anosovs are all obtained by this fibered Dehn filling from finitely many cusped hyperbolic 3-manifolds. Here, we focus on the smallest cusped manifold which has fibered Dehn fillings which are $\Sigma_{g}$-bundles with $g$ arbitrarily large, namely the Whitehead sibling. This is thus a very natural place to look for pseudo-Anosovs realizing the minimal dilatation $\delta_{g}$.

However, the idea of searching for pseudo-Anosovs with low dilatation among the monodromies of low-volume manifolds predates [5]. In particular, earlier Kin, Kojima and Takasawa had asked [14, Question 5.1]:

1.6 Question For a fixed surface $\Sigma$, possibly with punctures, is there a pseudoAnosov homeomorphism $\psi$ of $\Sigma$ which simultaneously minimizes $\lambda(\psi)$ and the volume of the mapping torus $M_{\psi}$ ?

There is good evidence that the answer is yes when $\Sigma$ is a punctured sphere [14; 24]. However, by combining our examples here with those of E Hironaka [12], we show in Theorem 7.5 that the answer is no for large $g \equiv 3 \bmod 5$, provided Conjecture 4.1 holds. However, we also show:

1.7 Theorem For large $g$, the monodromy of $M_{g}$ minimizes dilatation among all $\Sigma_{g}$-bundles obtained from filling $W$.

The proof uses McMullen's Teichmüller polynomial from [17] to systematically calculate and analyze the dilatation of all fillings of $W$. Independently, Kin and Takasawa [15] have also analyzed these fillings, obtaining similar results about dilatations (though not volumes) to those we describe here.

Let $\lambda_{g}$ be the dilatation of the monodromy of $M_{g}$ from Section 1.1. For $g \equiv 3 \bmod 5$, we show that $\lambda_{g}>\delta_{g}$. However, for $g \neq \equiv 3 \bmod 5$ and $g \neq 4$, the dilatations $\lambda_{g}$ seem 
to be the best known and in some cases better than previous examples. See Table 1 for the known examples in low genus. Very intriguingly, the dilatations of both our examples and those of [12] come from the same family of polynomials introduced by Lanneau and Thiffeault [16]:

$$
L T_{a, b}(t)=t^{2 a}-t^{a+b}-t^{a}-t^{a-b}+1 \quad \text { with } a>b .
$$

Both here and in [12], the parameter $a$ is within 2 of $g$ and $b$ is small.

\begin{tabular}{rrrrrrrr}
$g$ & \multicolumn{1}{c}{$\lambda_{g}$} & \multicolumn{1}{c}{$\epsilon_{g}$} & & $g$ & \multicolumn{1}{c}{$\lambda_{g}$} & \multicolumn{1}{c}{$\epsilon_{g}$} \\
\cline { 2 - 4 } 3 & $\circ 1.5061357$ & $\circ 1.4012684$ & & 21 & 1.0427679 & $\circ 1.0450879$ \\
4 & 1.2806382 & 1.2612310 & & 22 & 1.0425372 & 1.0430555 \\
5 & 1.1487947 & $\circ 1.1762808$ & & 23 & $\circ 1.0428751$ & $\circ 1.0409471$ \\
6 & 1.1287609 & 1.1617044 & & 24 & 1.0379320 & 1.0394979 \\
7 & $\circ 1.1154811$ & $\circ 1.1369400$ & & 25 & 1.0363101 & $\circ 1.0379320$ \\
8 & 1.1369400 & 1.1135007 & & 26 & 1.0349894 & 1.0363101 \\
9 & $\circ 1.0928247$ & $\circ 1.1054009$ & & 27 & $\circ 1.0338143$ & $\circ 1.0351470$ \\
10 & 1.0837668 & 1.0946555 & & 28 & 1.0351470 & 1.0339030 \\
11 & 1.0770457 & $\circ 1.0837668$ & & 29 & $\circ$ & 1.0315904 & $\circ 1.0326168$ \\
12 & 1.0726646 & 1.0787378 & & 30 & 1.0305456 & 1.0316628 \\
13 & $\circ 1.0776673$ & $\circ 1.0726646$ & & 31 & 1.0296057 & $\circ 1.0306505$ \\
14 & 1.0629875 & 1.0663959 & & 32 & 1.0288091 & 1.0296057 \\
15 & 1.0583358 & $\circ 1.0629875$ & & 33 & $\circ 1.0296414$ & $\circ 1.0288091$ \\
16 & 1.0549983 & 1.0590666 & & 34 & 1.0275530 & 1.0279692 \\
17 & $\circ 1.0522148$ & $\circ 1.0549983$ & & 35 & 1.0263611 & $\circ 1.0271037$ \\
18 & 1.0568996 & 1.0525403 & & 36 & 1.0256581 & 1.0264286 \\
19 & $\circ 1.0470846$ & $\circ 1.0497943$ & & 37 & $\circ 1.0250132$ & $\circ 1.0257204$ \\
\hline 20 & 1.0447582 & 1.0469430 & 38 & 1.0257204 & 1.0249917
\end{tabular}

Table 1: Low-dilatation pseudo-Anosovs. Here $\lambda_{g}$ is the dilatation associated to the manifold $M_{g}$, and $\epsilon_{g}$ is the dilatation of the examples in Theorem 1.3 of [12]. The darkened boxes have lower numbers, and $\circ$ indicates the invariant foliations are orientable.

However, asymptotically the $\lambda_{g}$ are no better than the previously known examples. A basic result of Penner says that $\log \delta_{g} \asymp 1 / g$ [21]. In that context, we show:

1.9 Theorem We have $\lim _{g \rightarrow \infty} \lambda_{g}^{g}=(3+\sqrt{5}) / 2=1+\gamma$, where $\gamma$ is the golden ratio.

The same result holds for the examples of [12] and also those of [15, Theorem 1.5]. 


\subsection{Orientable foliations}

A natural subclass of pseudo-Anosovs is the subclass whose invariant foliations are orientable. Analogously, define $\delta_{g}^{+}$to be the least dilatation among such maps. While $\delta_{g}$ is known only when $g \leq 2$ by Cho and Ham [3], the work of Lanneau and Thiffeault [16] and Hironaka [12] combine to determine $\delta_{g}^{+}$when $g \in\{2,3,4,5,8\}$. We add one more, showing:

1.11 Theorem The monodromy $\psi_{7}$ of the manifold $M_{7}$ minimizes dilatation among all pseudo-Anosovs of $\Sigma_{7}$ with orientable invariant foliations. In particular, one has $\delta_{7}^{+} \approx 1.11548110945659$.

Here the needed lower bound comes directly from [16], and Theorem 1.11 was independently established by [15]. See Table 2 for some of the known examples with orientable invariant foliations.

\begin{tabular}{rr|r}
$g$ & $\lambda_{g}^{+}$ & $\epsilon_{g}^{+}$ \\
\hline 3 & 1.5061357 & $* 1.4012684$ \\
5 & $* 1.1762808$ & $* 1.1762808$ \\
7 & $* 1.1154811$ & 1.1369400 \\
9 & 1.0928247 & 1.1054009 \\
11 & 1.0803146 & 1.0837668 \\
13 & 1.0776673 & 1.0726646 \\
15 & 1.0597294 & 1.0629875 \\
17 & 1.0522148 & 1.0549983 \\
19 & 1.0470846 & 1.0497943 \\
21 & 1.0433121 & 1.0450879 \\
23 & 1.0428751 & 1.0409471
\end{tabular}

\begin{tabular}{ccc}
$g$ & $\lambda_{g}^{+}$ & $\epsilon_{g}^{+}$ \\
\hline 25 & 1.0366424 & 1.0379320 \\
27 & 1.0338143 & 1.0351470 \\
29 & 1.0315904 & 1.0326168 \\
31 & 1.0297856 & 1.0306505 \\
33 & 1.0296414 & 1.0288091 \\
35 & 1.0264880 & 1.0271037 \\
37 & 1.0250132 & 1.0257204 \\
39 & 1.0237761 & 1.0244123 \\
41 & 1.0227200 & 1.0231851 \\
43 & 1.0226556 & 1.0221591 \\
45 & 1.0207537 & 1.0211819
\end{tabular}

Table 2: Pseudo-Anosovs with orientable invariant foliations. Here $\lambda_{g}^{+}$is the least dilatation of any example with orientable foliations obtained from $W$, and $\epsilon_{g}^{+}$is that of the examples in Theorem 1.4 of [12]. The manifold $W$ does not generate orientable examples when $g$ is even; however [12] provides such whenever $g \not \equiv 0 \bmod 6$. The darkened boxes have lower numbers, and a $*$ indicates the dilatation is known to be equal to $\delta_{g}^{+}$.

Acknowledgments We thank J O Button, Danny Calegari, Stefan Friedl, Toby Hall, A J Hildebrand, Eriko Hironaka, Eiko Kin, Sadayoshi Kojima, Erwan Lanneau, Chris Leininger, Curt McMullen, Shigeru Mizushima, Saul Schleimer, Mitsuhiko Takasawa and Jean-Luc Thiffeault for helpful conversations and correspondence. Dunfield was 
partially supported by the US NSF via grant DMS-0707136. The authors also thank the anonymous referee for very helpful comments and corrections, and Yuichi Kabaya for pointing out an error in the original version of Figure 1(b). The first author John W Aaber was formerly known as Rupert Venzke.

\section{The sibling of the Whitehead link complement}

This section gives a detailed picture of the 2-cusped hyperbolic 3-manifold that is the focus of this paper, namely the sibling of the Whitehead link complement. This manifold, which we denote by $W$, is the complement of the $(-2,3,8)$-pretzel link shown in Figure 3, and is manifold $m 125$ in the SnapPea census [2]. Like the Whitehead complement, it can be built by gluing the sides of a regular ideal octahedron in $\mathbb{H}^{3}$ (see [1]); thus its volume is

$$
V_{8}=4 \times(\text { Catalan's constant })=4\left(\sum_{k=0}^{\infty} \frac{(-1)^{k}}{(2 k+1)^{2}}\right) \approx 3.6638623767088 .
$$

SnapPea $[25 ; 4]$ finds that its fundamental group is

$$
\pi_{1}(W)=\langle x, y \mid y x y x Y x Y X Y X y X=1\rangle \text { where } X=x^{-1} \text { and } Y=y^{-1} \text {. }
$$

The defining relator actually comes from a handle decomposition of $W$, shown in Figure 1(a).

Notice that the relator is very symmetric in the following sense. Realize the free group on $\{x, y\}$ as the fundamental group of a wedge of two circles. Pass to the universal abelian cover and lift the relator as shown in Figure 2(a). From the picture, we see that both $\{x \mapsto y, y \mapsto x\}$ and $\{x \mapsto x, y \mapsto Y\}$ induce automorphisms of $\pi_{1}(W)$; together, they generate a subgroup of order 8 in $\operatorname{Out}\left(\pi_{1}(W)\right)$, which acts on $\pi_{1}(W)^{\mathrm{ab}}=H_{1}(W ; \mathbb{Z})=\mathbb{Z}^{2}$ as the dihedral group of the square. By Mostow-Prasad rigidity, these automorphisms come from isometries of $W$.

\subsection{Thurston norm}

Recall that the Thurston norm on $H^{1}(W ; \mathbb{R})$ organizes which cohomology classes correspond to fibrations over $S^{1}$ (throughout this subsection - see Thurston [23] for details). Before describing the norm in this case, we fix some notation. As a basis for $H_{1}(W ; \mathbb{Z})$, we use the images of the generators $\{x, y\}$ of $\pi_{1}(W)$ under abelianization map, denoted by the same symbols. However, we write the operation additively, eg $x^{2} y^{-3} \mapsto 2 x-3 y$. We'll use the dual basis $\left\{x^{*}, y^{*}\right\}$ for $H^{1}(W ; \mathbb{Z})$, and show: 


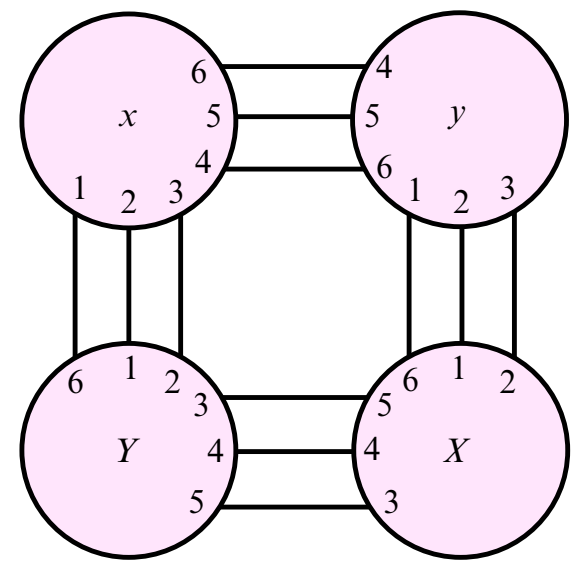

(a) A handle decomposition of $W$ : think of the above pattern on the boundary of a 3ball $B^{3}$, and glue the disks together to form a genus 2 handlebody. The arcs then form a single closed curve, along which you attach a 2-handle corresponding to the defining relator of $\pi_{1}(W)$.

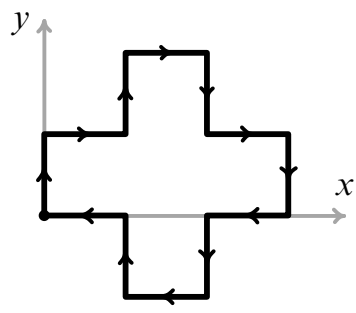

(a) The lifted relator

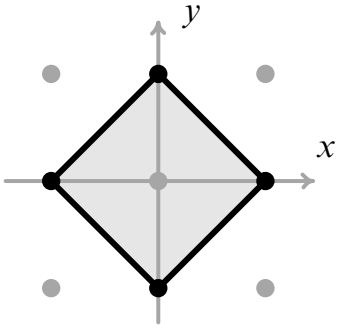

(b) The Newton polygon of $\Delta_{W}$ in $H_{1}(W ; \mathbb{Z})$

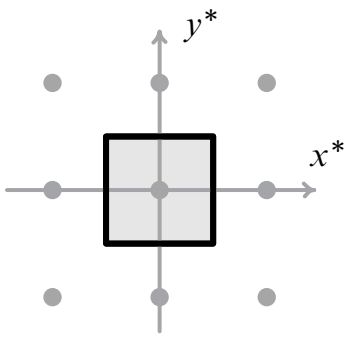

(c) The Thurston norm unit ball $B_{T} \subset H^{1}(W ; \mathbb{Z})$

Figure 2

2.2 Lemma The unit ball $B_{T}$ in the Thurston norm of $W$ is the square shown in Figure 2(c), and all four sides are fibered faces. In particular, every primitive element of $H^{1}(W ; \mathbb{Z})$ except for $\pm\left(x^{*} \pm y^{*}\right)$ gives rise to a fibration of $W$.

Proof We will use the Alexander norm introduced by McMullen [18] to compute the Thurston norm. From Figure 2(a), it is clear the Alexander polynomial of $W$, which 
lives in the group ring $\mathbb{Z}\left[H_{1}(W ; \mathbb{Z})\right]$, is

$$
\Delta_{W}=x^{-1}+x+1+y^{-1}+y,
$$

where the operation on $H_{1}(W ; \mathbb{Z})$ is written multiplicatively. The Newton polytope of $\Delta_{W}$ is shown in Figure 2(b); hence the unit ball $B_{A}$ of the Alexander norm, which is its dual, is as in Figure 2(c). Since $b_{1}(W)>1$, we have that $B_{T} \subset B_{A}$ [18]. To complete the proof we need to show that $B_{T}=B_{A}$ and all faces (sides) of $B_{T}$ are fibered. By the convexity of $B_{T}$ and the symmetries of $W$ noted above, it is enough to show:

(1) The Thurston norm $\left\|x^{*}+y^{*}\right\| \leq 2$.

(2) The manifold $W$ fibers over the circle.

For (1), we simply exhibit a twice-punctured torus dual to $x^{*}+y^{*}$ in Figure 1(b). For (2), Figure 3 shows that $W$ fibers over the circle with fiber a 5-punctured disc, with corresponding monodromy $\psi=\sigma_{1}^{3} \sigma_{2} \sigma_{3} \sigma_{4}$.

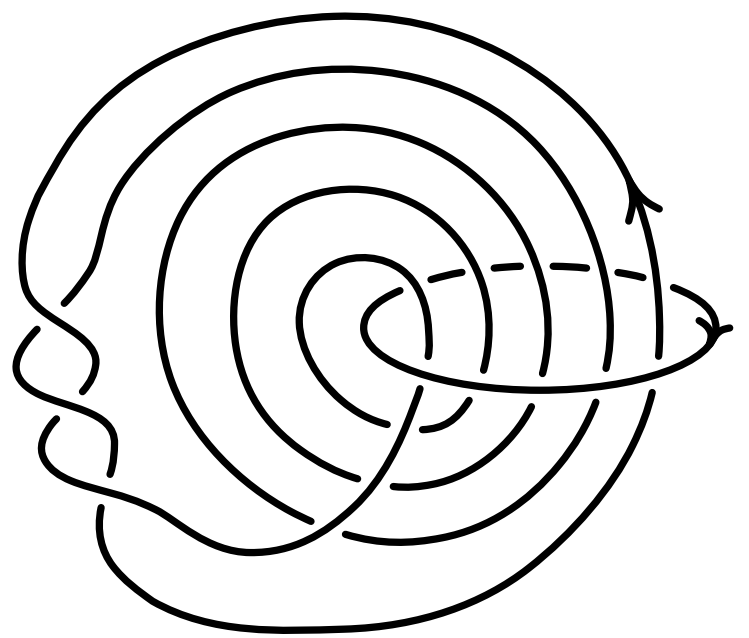

Figure 3: The manifold $W$ is the exterior of the $(-2,3,8)-$ pretzel link, shown here in a nonstandard projection exhibiting the fibration of $W$ corresponding to $2 x^{*}+y^{*}$. In terms of the usual homological framing on each component, we have $\mu_{1}=(-7,1), \lambda_{1}=(1,0)$ and $\mu_{2}=(-4,1), \lambda_{2}=$ $(-3,1)$, where the unknotted component corresponds to the second cusp. Our orientation conventions are given by $\boldsymbol{W}$, and match those of SnapPea [25; 4]. That the fibration corresponds to $2 x^{*}+y^{*}$ follows since the meridian of the knotted component is $\lambda_{1}=-x+2 y$ by (2.5). 


\subsection{Dehn filling}

Think of $W$ as a compact manifold having two torus boundary components $\partial_{1} W$ and $\partial_{2} W$. On each boundary component, we use the basis $\left(\mu_{k}, \lambda_{k}\right)$ of $H_{1}\left(\partial_{k} W ; \mathbb{Z}\right)$ defined in Figure 3. The shape of each cusp in the hyperbolic structure on $\operatorname{int}(W)$ with respect to $\left(\mu_{k}, \lambda_{k}\right)$ is a Euclidean square. For either component, we have that the image of $H_{1}\left(\partial_{k} W ; \mathbb{Z}\right) \rightarrow H_{1}(W ; \mathbb{Z}) \cong \mathbb{Z}^{2}$ is a sublattice of index 5; explicitly, SnapPea finds that

$$
\left(\mu_{1}, \lambda_{1}\right) \mapsto(2 x+y,-x+2 y) \quad \text { and } \quad\left(\mu_{2}, \lambda_{2}\right) \mapsto(x+2 y, 2 x-y) .
$$

2.6 Remark This may seem like a minor point, but the fact $H_{1}\left(\partial_{k} W ; \mathbb{Z}\right)$ is nearly all of $H_{1}(W ; \mathbb{Z})$ is what distinguishes this case from that of the Whitehead complement, where the image is merely $\mathbb{Z}$.

Now given any $\phi \in H^{1}(W ; \mathbb{Z})$, there is a unique simple closed curve in the kernel of $\phi: H_{1}\left(\partial_{k} W\right) \rightarrow \mathbb{Z}$. Thus, there is a unique Dehn filling on $W$ yielding a closed manifold $W_{\phi}$ so that $\phi$ extends to a map $H_{1}\left(W_{\phi}\right) \rightarrow \mathbb{Z}$. Dually, if $F_{\phi}$ is a surface dual to $\phi$, then $W_{\phi}$ is the Dehn filling of $W$ where the components of $\partial F$ bound discs in the attached solid tori.

We are interested in those primitive $\phi \in H^{1}(W ; \mathbb{Z})$ corresponding to fibrations. Because of the symmetries of $W$ noted above, we will restrict to $\phi=a x^{*}+b y^{*}$ where $0 \leq b<a$ and $\operatorname{gcd}(a, b)=1$. Because $H_{1}\left(\partial_{k} W ; \mathbb{Z}\right)$ has index 5 in $H_{1}(W ; \mathbb{Z})$, there are various small repeating issues throughout this paper, requiring us to define:

$$
\epsilon(\phi)= \begin{cases}1 & \text { if } a^{2}+b^{2} \equiv 0 \bmod 5 \\ 0 & \text { otherwise }\end{cases}
$$

$\epsilon_{1}(\phi)=\left\{\begin{array}{ll}1 & \text { if } 2 a+b \equiv 0 \bmod 5, \\ 0 & \text { otherwise }\end{array} \quad\right.$ and $\quad \epsilon_{2}(\phi)= \begin{cases}1 & \text { if } a+2 b \equiv 0 \bmod 5 \\ 0 & \text { otherwise }\end{cases}$

These quantities are related by:

2.7 Lemma Suppose $\operatorname{gcd}(a, b)=1$. Then $\epsilon(\phi)=\epsilon_{1}(\phi)+\epsilon_{2}(\phi)$.

Proof First notice that one never has $\epsilon_{1}(\phi)=\epsilon_{2}(\phi)=1$, as then $a \equiv b \equiv 0 \bmod 5$, since $\left(\begin{array}{ll}2 & 1 \\ 1 & 2\end{array}\right)$ is invertible over $\mathbb{F}_{5}$. The result then follows from the observation that $(2 a+b)(a+2 b) \equiv 2\left(a^{2}+b^{2}\right) \bmod 5$.

2.8 Theorem Let $\phi=a x^{*}+b y^{*}$ where $0 \leq b<a$ and $\operatorname{gcd}(a, b)=1$. There is a unique fibration of $W_{\phi}$ over the circle, and the genus of the fiber is $a-2 \epsilon(\phi)$. 
Proof By Lemma 2.2, the Thurston norm ball $B_{T}$ for $W$ is the square pictured in Figure 2(c). Our $\phi$ lies over the righthand face of $B_{T}$ where $x^{*}=1 / 2$, and so $\|\phi\|=2 a$. As all the faces of $B_{T}$ are fibered, the class $\phi$ gives a fibration of $W$ over the circle where the fiber $F$ has Euler characteristic $-\|\phi\|=-2 a$. Let $F_{\phi}$ be the corresponding closed surface in $W_{\phi}$. Then $\chi\left(F_{\phi}\right)=\chi(F)+d_{1}+d_{2}$, where $d_{k}$ is the number of boundary components of $F$ on $\partial_{k} W$. An easy calculation using Lemma 2.7 shows that the claim

$$
d_{k}=1+4 \epsilon_{k}(\phi)
$$

implies that the genus of $F_{\phi}$ is $a-2 \epsilon(\phi)$.

To complete the proof, we establish (2.9), focusing on the case when $k=1$ as the other is similar. Using (2.5), we have $d_{1}=\operatorname{gcd}\left(\phi\left(\mu_{1}\right), \phi\left(\lambda_{1}\right)\right)=\operatorname{gcd}(2 a+b,-a+2 b)=$ $\operatorname{gcd}(2 a+b,-5 b)$, which is 5 if $2 a+b \equiv 0 \bmod 5$ and 1 otherwise. (Geometrically, these are the only two possibilities because $\left[H_{1}(W ; \mathbb{Z}): H_{1}\left(\partial_{1} W ; \mathbb{Z}\right)\right]=5$.) That is, $d_{1}=1+4 \epsilon_{1}(\phi)$ as needed.

For future reference, we record the precise Dehn filling description of $W_{\phi}$.

2.10 Lemma Let $\phi=a x^{*}+b y^{*}$ where $0 \leq b<a$ and $\operatorname{gcd}(a, b)=1$. Then $W_{\phi}$ is the Dehn filling on $W$ given by $W((a-2 b) /(2 a+b),(-2 a+b) /(a+2 b))$. More precisely, the filling is along the homology classes

$$
\begin{aligned}
& \gamma_{1}=5^{-\epsilon_{1}(\phi)}\left((a-2 b) \mu_{1}+(2 a+b) \lambda_{1}\right) \\
& \gamma_{2}=5^{-\epsilon_{2}(\phi)}\left((-2 a+b) \mu_{2}+(a+2 b) \lambda_{2}\right) .
\end{aligned}
$$

Proof The surgery curve $\gamma_{k}$ is a primitive class killed by $\phi: H_{1}\left(\partial_{k} W ; \mathbb{Z}\right) \rightarrow \mathbb{Z}$. Now one element in the kernel is $-\phi\left(\lambda_{k}\right) \mu_{k}+\phi\left(\mu_{k}\right) \lambda_{k}$, and as noted in the proof of Theorem 2.8, we have $\operatorname{gcd}\left(\phi\left(\mu_{k}\right), \phi\left(\lambda_{k}\right)\right)=1+4 \epsilon_{k}(\phi)=5^{\epsilon_{k}(\phi)}$. Thus

$$
\gamma_{k}=5^{-\epsilon_{k}(\phi)}\left(-\phi\left(\lambda_{k}\right) \mu_{k}+\phi\left(\mu_{k}\right) \lambda_{k}\right)
$$

is a primitive element of $H_{1}\left(\partial_{k} W ; \mathbb{Z}\right)$, and expanding using (2.5) gives the claimed formulae.

\section{Examples with a given genus}

For a typical fixed genus $g$, there are many different fillings on $W$ which are $\Sigma_{g}-$ bundles. In this section, we organize these manifolds and define our main examples $M_{g}$, which have the smallest volume among all $\Sigma_{g}$-bundles arising from $W$ (at least 
when $g$ is large). As in the previous section, by symmetry we consider only those $\phi \in H^{1}(W ; \mathbb{Z})$ of the form $\phi=a x^{*}+b y^{*}$ with $0 \leq b<a$ and $\operatorname{gcd}(a, b)=1$. We also assume that $g>2$, as the manifolds arising for $g \leq 2$ are not hyperbolic. By Theorem 2.8, the $\phi$ for which $W_{\phi}$ is a $\Sigma_{g}$-bundle fall into two classes

$$
\begin{aligned}
\mathcal{A}_{g} & =\left\{\phi=g x^{*}+b y^{*} \mid \epsilon(\phi)=0, \operatorname{gcd}(g, b)=1\right\} \\
\mathcal{E}_{g}=\{\phi & \left.=(g+2) x^{*}+b y^{*} \mid \epsilon(\phi)=1, \operatorname{gcd}(g+2, b)=1\right\} .
\end{aligned}
$$

We show below:

3.2 Proposition For each $g>2$, the following holds:

(1) The set $\mathcal{A}_{g}$ is nonempty.

(2) The set $\mathcal{E}_{g}$ is nonempty if and only if $g \not \equiv 3 \bmod 5$ and $g>4$.

Section 5 shows that manifolds coming from $\mathcal{E}_{g}$ typically have smaller volume than those coming from $\mathcal{A}_{g}$, and volume increases as $b$ increases. Hence we define:

3.3 Definition For each $g>2$, if $\mathcal{E}_{g}$ is nonempty, we define $M_{g}$ to be $W_{\phi}$ where $\phi \in \mathcal{E}_{g}$ is the class where $b$ is smallest. Otherwise, $M_{g}$ comes from the $\phi \in \mathcal{A}_{g}$ where again $b$ is smallest.

For large $g$, we will show in Theorem 5.1 that $M_{g}$ indeed minimizes volume among all $\Sigma_{g}$-bundles arising from $W$. In the rest of this section, we first prove Proposition 3.2, and then refine it to give the precise Dehn filling description of each $M_{g}$.

Proof of Proposition 3.2 We begin with part (1). If $g \equiv 0,1,4 \bmod 5$, then $\phi=$ $g x^{*}+y^{*}$ is an element of $\mathcal{A}_{g}$. So suppose instead that $g \equiv 2,3 \bmod 5$. Now $\mathcal{A}_{3}=\left\{3 x^{*}+2 y^{*}\right\}$, so we can assume that $g \geq 7$. Then $g x^{*}+5 y^{*} \in \mathcal{A}_{g}$, completing the proof of (1).

For part (2), first suppose $g \equiv 3 \bmod 5$. If $\phi=(g+2) x^{*}+b y^{*}$ is in $\mathcal{E}_{g}$, the requirement that $\epsilon(\phi)=1$ forces $b^{2} \equiv 0 \bmod 5$. But then $g+2$ and $b$ are both divisible by 5 , a contradiction. So $\mathcal{E}_{g}$ is empty in this case. If $g \equiv 0,1 \bmod 5$, then $\phi=(g+2) x^{*}+y^{*}$ is in $\mathcal{E}_{g}$, and if $g \equiv 2 \bmod 5$, we may simply take $b=g+1$.

So we've reduced to when $g \equiv 4 \bmod 5$. Now $\mathcal{E}_{4}$ is empty, so assume $g>4$, in which case $a=g+2 \geq 11$. If $a$ is coprime to either 2 or 3 , we simply use that for $b$, so assume $a=2^{c} 3^{d} L$ where $c, d \geq 1$ and $L$ is coprime to 6 . If $L=1$, we take $b=7$. Thus we can assume that $L>1$, which forces $L \geq 7$ as $a$ is coprime to 5 .

When $L \equiv 1 \bmod 3$, we will select $b$ from among $\{L+4, L+6, L+10, L+12\}$, which are all less than $a \geq 6 L$ since $L \geq 7$. Each such number is coprime to 2,3 and $L$ 
(for $L+10$ this is because $5 \nmid L$ ), and hence to $a$. As they take on four distinct values modulo 5, at least one is $\equiv 2,3 \bmod 5$ giving the needed $b$. If instead $L \equiv 2 \bmod 3$, the same reasoning shows that we can choose $b$ from among $\{L+2, L+6, L+8, L+24\}$.

A more detailed description is:

3.4 Proposition Let $\phi=a x^{*}+b y^{*}$ be the class defining $M_{g}$.

(1) If $g \equiv 0,1 \bmod 5$, then $(a, b)=(g+2,1)$.

(2) If $g \equiv 3 \bmod 5$, then

(a) $(a, b)=(g, 2)$ when $g \equiv 3,13,23 \bmod 30$.

(b) $(a, b)=(g, 3)$ when $g \equiv 8,28 \bmod 30$.

(c) $(a, b)=(g, 5)$ when $g \equiv 18 \bmod 30$.

(3) If $g \equiv 2,4 \bmod 5$ with $g>4$ then $a=g+2$ and $b$ is the smallest number coprime to $g+2$ satisfying $b \equiv 2,3 \bmod 5$.

(4) If $g=4$, then $(a, b)=(4,1)$.

(5) For any $g$, the coefficient $b$ is $O(\log g)$ and is either 1 or a prime.

Proof Everything is immediate from Proposition 3.2 with the exception of (2) and (5). For the first, assume $g \equiv 3 \bmod 5$ is the element of $\mathcal{A}_{g}$ for which $b$ is minimal. Now $g$ is coprime to 5 , and so $b$ is at most 5 ; since we need $\epsilon(\phi)=0$, that is $g^{2}+b^{2} \not \equiv 0 \bmod 5$, this means $b \in\{2,3,5\}$. Which of these satisfy $\operatorname{gcd}(g, b)=1$ can be determined by the class of $b$ modulo 30, giving the statement above.

The claim (5) is clear in all cases except (3), so assume that $g \equiv 2,4 \bmod 5$ and $b$ is the smallest number coprime to $a=g+2$ satisfying $b \equiv 2,3 \bmod 5$. Note that $b$ is necessarily prime since, if not, it would have a prime factor which is $\equiv 2,3 \bmod 5$ which would also be coprime to $a$. The following argument, which we learned from A J Hildebrand, shows that $b$ is $O(\log g)$.

Call a prime $\operatorname{good}$ if it is $\equiv 2,3 \bmod 5$. By minimality of $b$, every power $p^{k}<b$ of a good prime must divide $a$. Thus

$$
a \geq \prod\left\{p^{k} \mid p \text { a good prime and } k \text { maximal with } p^{k}<b\right\} .
$$

Taking logs and then applying the prime number theorem for arithmetic progressions, we get

$$
\log (a) \geq \sum_{\substack{p^{k}<b, p \text { good }}} \log p=\psi(b ; 5,2)+\psi(b ; 5,3)=\frac{b}{2}+O\left(b e^{-c \sqrt{\log b}}\right)=b\left(\frac{1}{2}+o(1)\right)
$$


where the terms in the center are Chebyshev's $\psi$-function, and $c>0$ is an unknown constant (see eg [13, Corollary 2 on page 138]). Hence $b \leq(2+o(1)) \log (a)$ as needed.

As in Section 2, let $W\left(p_{1} / q_{1}, p_{2} / q_{2}\right)$ denote the manifold obtained by performing Dehn filling on $W$. Combining Proposition 3.4 and Lemma 2.10, we could just as well have defined $M_{g}$ for $g>2$ via the following:

(1) If $g \equiv 0,1 \bmod 5$, then $M_{g}=W((g) /(2 g+5),-(2 g+3) /(g+4))$.

(2) If $g \equiv 3 \bmod 5$, then

(a) $M_{g}=W((g-4) /(2 g+2),(2-2 g) /(g+4))$ when $g \equiv 3,13,23 \bmod 30$.

(b) $M_{g}=W((g-6) /(2 g+3),(3-2 g) /(g+6))$ when $g \equiv 8,28 \bmod 30$.

(c) $M_{g}=W((g-10) /(2 g+5),(5-2 g) /(g+10))$ when $g \equiv 18 \bmod 30$.

(3) If $g \equiv 2,4 \bmod 5$ and $g>4$, then $M_{g}=W((-2 b+g+2) /(b+2 g+4)$, $(b-2(g+2)) /(2 b+g+2))$, where $b$ is the smallest number coprime to $g+2$ satisfying $b \equiv 2,3 \bmod 5$.

(4) $M_{4}=W(2 / 9,-7 / 6)$.

\section{Proof of the basic theorem}

Our claim that the $M_{g}$ are the smallest volume genus- $g$ bundles for large $g$ is conditional on the following conjecture:

4.1 Conjecture Suppose $M$ is a closed hyperbolic 3-manifold with $b_{1}(M) \geq 2$. Then $\operatorname{Vol}(M) \geq V_{8} \approx 3.663862$.

This conjecture is very plausible for two reasons. First, the smallest example of this type known has volume $\geq 4.71$. (The example is $M=9_{4}^{2}(0,1)(0,1)=v 1539(5,1)=$ $s 941(5,1)(5,1)$ and has the least volume among all 0 -surgeries on homologically split 2-component links with $<15$ crossings, as well as the manifolds in (or obtained from) certain standard censuses $[2 ; 10]$.) Second, if we broaden the context from closed to finite-volume, experimentally it seems that the smallest volume hyperbolic manifold with $b_{1}(M) \geq n$ always has cusps (see [7, Section 10]), and Conjecture 4.1 is known for cusped manifolds:

4.2 Theorem Let $M$ be a finite-volume hyperbolic 3-manifold with at least one cusp. If $b_{1}(M) \geq 2$, then $\operatorname{Vol}(M) \geq V_{8}$ with equality exactly when $M$ is the Whitehead complement or its sibling. 
Theorem 4.2 is essentially proved in [1], though we give the details below. Understanding the volumes of closed manifolds is fundamentally harder than that of cusped manifolds, so Conjecture 4.1 seems well beyond present technology (see [7] for a survey). It is worth noting that, by arguments similar to those proving Theorem 4.3, there are at most finitely many counterexamples to Conjecture 4.1 .

Proof of Theorem 4.2 Suppose $M$ has at least two cusps; then the conclusion is exactly Theorem 3.6 of [1]. So suppose $M$ has one cusp. Since

$$
b_{1}(M)=\operatorname{rank} H^{1}(M ; \mathbb{Z})=\operatorname{rank} H_{2}(M, \partial M ; \mathbb{Z}) \geq 2,
$$

we must have that $H_{2}(M ; \mathbb{Z}) \neq 0$. Let $\Sigma$ be a closed incompressible surface representing a nonzero class in $H_{2}(M ; \mathbb{Z})$. Since it is disjoint from the cusp, Theorem 3.5 of [1] gives that either $\operatorname{Vol}(M)>V_{8}$ or $M$ is obtained by identifying the sides of a regular ideal octahedron. The latter case gives finitely many possibilities, none of which have one cusp and $b_{1}(M) \geq 2$. One can either check this by hand, or just use that such manifolds have been independently enumerated in [2] and [11].

We can now prove the basic version of the main result of this paper.

4.3 Theorem If Conjecture 4.1 is true, then for every large $g$ the hyperbolic $\Sigma_{g}-$ bundle of minimal volume is a Dehn filling on the sibling $W$ of the Whitehead complement.

Proof Suppose to the contrary that there is an infinite sequence $\left(N_{n}, g_{n}\right)$, where each $N_{n}$ is a minimal volume $\Sigma_{g_{n}}$-bundle which is not a Dehn filling of $W$. Here $g_{n} \rightarrow \infty$ as $n \rightarrow \infty$ since there are finitely many manifolds of any given volume and hence finitely many minimal volume $\Sigma_{g}$-bundles for each fixed $g$. From Section 3, we know that for each $g>2$ there exists a $\Sigma_{g}$-bundle $M_{g}$ which is a Dehn filling on $W$. Since volume strictly decreases under Dehn filling, we have $\operatorname{Vol}\left(M_{g}\right)<\operatorname{Vol}(W)=V_{8}$ for all $g$, and hence by minimality that $\operatorname{Vol}\left(N_{n}\right)<V_{8}$ for all $n$. As their volumes are bounded, we pass to a subsequence where the $N_{n}$ have a geometric limit $N$; for ease of notation, we reindex so that $N_{n}$ now denotes that subsequence. As $N$ is the geometric limit of the $N_{n}$, we have $\operatorname{Vol}(N)=\lim _{n \rightarrow \infty} \operatorname{Vol}\left(N_{n}\right)$ and so $\operatorname{Vol}(N) \leq V_{8}$. There are three cases, depending on how many cusps $N$ has.

Case 1 (No cusps) If $N$ is compact, then the sequence is eventually constant, with $N_{n} \cong N$ for all large $n$. As $g_{n} \rightarrow \infty$, the manifold $N$ must fiber in infinitely many ways, in particular $b_{1}(N) \geq 2$. But then Conjecture 4.1 implies that $\operatorname{Vol}(N) \geq V_{8}$, a contradiction since $\operatorname{Vol}\left(N_{n}\right)<V_{8}$ for all $n$. 
Case 2 (One cusp) Suppose $N$ has one cusp. For all but one Dehn filling of $N$, the first betti number of the filled manifold is $b_{1}(N)-1$. (The exception is the filling along the kernel of $H_{1}(\partial N ; \mathbb{Q}) \rightarrow H_{1}(N ; \mathbb{Q})$.) Thus since $b_{1}\left(N_{n}\right) \geq 1$ for every $n$, we have $b_{1}(N) \geq 2$. But then Theorem 4.2 implies that $\operatorname{Vol}(N)>V_{8}$, a contradiction to our observation that $\operatorname{Vol}(N) \leq V_{8}$.

Case 3 (At least 2 cusps) As noted, we have $\operatorname{Vol}(N) \leq V_{8}$. Since $N$ has at least 2 cusps, we know $b_{1}(N) \geq 2$ and so Theorem 4.2 implies that $N$ is either the complement of the Whitehead link or its sibling. Our assumption on the $N_{n}$ means it must be the former, which we denote $W^{\prime}$. For each of the two boundary components $\partial_{i} W^{\prime}$, there is a natural meridian-longitude pair $\left(\mu_{i}, \lambda_{i}\right)$ in $H_{1}\left(\partial_{i} W^{\prime} ; \mathbb{Z}\right)$. Here, $H_{1}\left(W^{\prime} ; \mathbb{Z}\right) \cong$ $\mathbb{Z}^{2}=\left\langle\mu_{1}, \mu_{2}\right\rangle$ and both $\lambda_{i}=0$ in $H_{1}\left(W^{\prime} ; \mathbb{Z}\right)$. Now each $H^{1}\left(N_{n} ; \mathbb{Z}\right) \neq 0$, and so gives rise to a nonzero class $\phi_{n} \in H^{1}\left(W^{\prime} ; \mathbb{Z}\right)$. The Dehn filling curve on $\partial_{1} W^{\prime}$ lies in the kernel of $\phi_{n}$, and thus either is $\lambda_{1}$ or $\phi_{n}=\mu_{2}^{*}$. Since the $N_{n}$ geometrically limit on $W^{\prime}$, the Dehn filling curves must be varying on both cusps, forcing $\phi_{n}=\mu_{2}^{*}$ for all but finitely many $n$. But the symmetric argument with the other cusp shows that $\phi_{n}=\mu_{1}^{*}$ for all but finitely many $n$, a contradiction.

In each case we have a contradiction, and thus all but finitely many of the $N_{n}$ must be fillings of the Whitehead sibling $W$.

\section{Volumes of Dehn fillings}

Since they are Dehn fillings, each $W_{\phi}$ has smaller volume than $W$, and most of them have volumes very close to $\operatorname{Vol}(W)$. In this section, we give very precise estimates on the volumes of most $W_{\phi}$, allowing us to show:

5.1 Theorem For all large $g$, the manifold $M_{g}$ minimizes volume among all $\Sigma_{g}-$ bundles which are Dehn fillings on the sibling of the Whitehead complement.

Together with Theorem 4.3, this will prove Theorem 1.2.

Throughout this section, we consider $\phi=a x^{*}+b y^{*} \in H^{1}(W ; \mathbb{Z})$ with $0 \leq b<a$ and $\operatorname{gcd}(a, b)=1$. The key quantity controlling $\operatorname{Vol}\left(W_{\phi}\right)$ turns out to be

$$
n(\phi)=\frac{5\left(a^{2}+b^{2}\right)}{1+25^{\epsilon(\phi)}} .
$$

As a first step, we use work of Neumann and Zagier [19] to prove:

\subsection{Lemma}

$$
\operatorname{Vol}\left(W_{\phi}\right)=\operatorname{Vol}(W)-\frac{\pi^{2}}{n(\phi)}+O\left(\frac{1}{n(\phi)^{2}}\right)
$$


This lemma suggests that $n(\phi)>n(\psi)$ implies $\operatorname{Vol}\left(W_{\phi}\right)>\operatorname{Vol}\left(W_{\psi}\right)$, which, if true, easily implies Theorem 5.1 given the formula for $n(\phi)$. However, we will need to refine the lemma substantially in order to actually prove the theorem.

Proof of Lemma 5.2 For any finite-volume hyperbolic 3-manifold $M$, Neumann and Zagier proved the following asymptotic formula for the volumes of large Dehn fillings. Let $T_{k}$ be a Euclidean torus associated to the $k$-th cusp, and let $\gamma_{k}=p_{k} \mu_{k}+q_{k} \lambda_{k}$ be the Dehn filling curve for that cusp. In terms of the quadratic form

$$
Q\left(\gamma_{k}\right)=Q\left(p_{k}, q_{k}\right)=\left(\text { length of } \gamma_{k}\right)^{2} /\left(\text { area of } T_{k}\right),
$$

Theorem 1A of [19] states

(5.3)

$$
\operatorname{Vol}\left(M \text { filled along the } \gamma_{i}\right)=\operatorname{Vol}(M)-\pi^{2} \sum_{k} \frac{1}{Q\left(p_{k}, q_{k}\right)}+O\left(\sum_{k} \frac{1}{p_{k}^{4}+q_{k}^{4}}\right) \text {. }
$$

As mentioned in Section 2.4, for $W$ with the bases we've chosen, the $T_{k}$ are both squares with sides parallel to $\mu_{k}$ and $\lambda_{k}$. From Lemma 2.10, one finds $Q\left(\gamma_{k}\right)=$ $5^{1-2 \epsilon_{k}(\phi)}\left(a^{2}+b^{2}\right)$. Using Lemma 2.7, we see that the first sum in (5.3) is simply $1 / n(\phi)$ as claimed. As for the error term, just observe that because of the square cusp shape one has $p_{k}^{4}+q_{k}^{4} \leq\left(p_{k}^{2}+q_{k}^{2}\right)^{2}=Q\left(\gamma_{k}\right)^{2}$ and so

$$
\sum_{k} \frac{1}{p_{k}^{4}+q_{k}^{4}} \leq \sum_{k} \frac{1}{Q\left(\gamma_{k}\right)}=\frac{1+5^{4 \epsilon}}{25\left(a^{2}+b^{2}\right)^{2}} \leq \frac{1}{n(\phi)^{2}}
$$

and hence we can view the error in (5.3) as $O\left(n(\phi)^{-2}\right)$.

Lemma 5.2 is enough to distinguish between the two classes of examples introduced in (3.1).

5.4 Theorem For all large $g$, if $\phi \in \mathcal{A}_{g}$ and $\psi \in \mathcal{E}_{g}$ then $\operatorname{Vol}\left(W_{\phi}\right)>\operatorname{Vol}\left(W_{\psi}\right)$.

Proof Notice that

$$
\frac{n(\phi)}{n(\psi)}=\frac{(5 / 2)\left(g^{2}+b_{1}^{2}\right)}{(5 / 26)\left((g+2)^{2}+b_{2}^{2}\right)}=13 \frac{g^{2}+b_{1}^{2}}{(g+2)^{2}+b_{2}^{2}}>\frac{13}{2}\left(\frac{g}{g+2}\right)^{2}
$$

and thus $n(\phi) \geq 6 n(\psi)$ for large $g$. Hence

$$
\operatorname{Vol}\left(W_{\phi}\right)-\operatorname{Vol}\left(W_{\psi}\right)=\pi^{2}\left(\frac{1}{n(\psi)}-\frac{1}{n(\phi)}\right)+O\left(\frac{1}{n(\phi)^{2}}\right) \geq \frac{5 \pi^{2}}{n(\phi)}+O\left(\frac{1}{n(\phi)^{2}}\right)
$$

which is positive for large $n(\phi)$, and hence for large $g$, as desired. 
Unfortunately, Lemma 5.2 is not enough to identify the minimal volume manifold among those coming from $\mathcal{A}_{g}$ (or $\mathcal{E}_{g}$ ). As a concrete example, if $g \equiv 13 \bmod 30$, then $\phi=g x^{*}+2 y^{*}$ and $\psi=g x^{*}+3 y^{*}$ are both in $\mathcal{A}_{g}$. However, the difference in volumes is governed by $1 / n(\psi)-1 / n(\phi)=2 /(n(\phi) n(\psi))$, which is comparable to the error. To distinguish such examples, we use that $\phi$ and $\psi$ are very closely related (in this case, $b$ differs by 1 ) to show that the error terms in Lemma 5.2 are also correlated, and this difference is accurate to one higher order than the original estimate itself. To execute this we need the following refined volume estimate.

5.5 Theorem Consider $\phi=a x^{*}+b y^{*} \in H^{1}(W ; \mathbb{Z})$ with $0 \leq b<a$ and $\operatorname{gcd}(a, b)=1$. If $\epsilon(\phi)=0$ then

$$
\operatorname{Vol}\left(W_{\phi}\right)=\operatorname{Vol}(W)-\frac{2 \pi^{2}}{5\left(a^{2}+b^{2}\right)}-\frac{\pi^{4}\left(7 a^{4}+144 a^{2} b^{2}+7 b^{4}\right)}{1875\left(a^{2}+b^{2}\right)^{4}}+O\left(\frac{1}{a^{6}}\right) .
$$

If instead $\epsilon(\phi)=1$ then $\operatorname{Vol}\left(W_{\phi}\right)-\operatorname{Vol}(W)$ is given by

$$
\begin{aligned}
& -\frac{26 \pi^{2}}{5\left(a^{2}+b^{2}\right)} \\
& \quad+\frac{\pi^{4}\left(901\left(a^{4}+b^{4}\right)+(-1)^{\epsilon_{1}(\phi)} 2808\left(a b^{3}-a^{3} b\right)-6336 a^{2} b^{2}\right)}{375\left(a^{2}+b^{2}\right)^{4}}+O\left(\frac{1}{a^{6}}\right) .
\end{aligned}
$$

Proof We will derive a more refined asymptotic expansion for $\operatorname{Vol}\left(W_{\phi}\right)$ using the method of [19]. For the standard triangulation of $W=m 125$ with four tetrahedra, the gluing equations are

$$
z_{1}\left(1-z_{2}\right)\left(1-z_{3}\right)\left(1-z_{4}\right)=-\left(1-z_{1}\right) \text { and }\left(1-z_{1}\right) z_{2} z_{4}=z_{3}
$$

where $z_{i} \in \mathbb{C}$ is the shape of the $i$-th tetrahedron. A solution to these equations gives a (typically incomplete) hyperbolic structure on $W$, with the induced holonomy $h$ on the boundary given by

$$
\begin{array}{ll}
U_{1}=h\left(\mu_{1}\right)=\frac{1}{z_{1}\left(1-z_{1}\right) z_{2} z_{4}\left(1-z_{4}\right)}, & V_{1}=h\left(-\lambda_{1}\right)=-\frac{z_{2}\left(1-z_{3}\right)\left(1-z_{4}\right)}{z_{4}}, \\
U_{2}=h\left(\mu_{2}\right)=-\frac{\left(1-z_{1}\right) z_{3}}{\left(1-z_{3}\right)\left(1-z_{4}\right)}, & V_{2}=h\left(-\lambda_{2}\right)=\frac{z_{1} z_{2}\left(1-z_{4}\right)}{\left(1-z_{1}\right) z_{4}} .
\end{array}
$$

Here, to match the conventions of [19], we are using bases $\left(\mu_{k},-\lambda_{k}\right)$ for the homology of the $k$-th cusp.

Following [19], we consider $u_{k}=\log U_{k}$ and $v_{k}=\log V_{k}$. On a neighborhood of $\mathbf{0} \in \mathbb{C}^{2}$, the pair $\mathbf{u}=\left(u_{1}, u_{2}\right)$ parameterize solutions to the gluing equations near the 
complete hyperbolic structure. The $v_{k}$ can be related to the $u_{k}$ via a potential function $\Phi(\mathbf{u})$, where in particular

$$
v_{k}=\frac{1}{2} \frac{\partial \Phi}{\partial u_{k}} .
$$

Because there is an isometry of $W$ interchanging $\left(\mu_{1}, \lambda_{1}\right)$ with $\left(\mu_{2}, \lambda_{2}\right)$, the potential function has the symmetric form

$$
\Phi(\mathbf{u})=c_{1}\left(u_{1}^{2}+u_{2}^{2}\right)+c_{2}\left(u_{1}^{4}+u_{2}^{4}\right)+c_{3} u_{1}^{2} u_{2}^{2}+O\left(|\mathbf{u}|^{6}\right) .
$$

The function $\Phi$ also determines the following refined volume estimate of [19]. If $W_{\mathbf{u}}$ denotes the hyperbolic structure on $W$ corresponding to $\mathbf{u}$, and $\Phi_{k}$ the terms of $\Phi$ of total degree $k$, then

$$
\operatorname{Vol}\left(W_{\mathbf{u}}\right)=\operatorname{Vol}(W)+\frac{1}{4} \cdot \operatorname{Im}\left(\sum_{k=1}^{2} u_{k} \bar{v}_{k}-\frac{1}{2} \sum_{k=2}^{\infty}(k-2) \Phi_{k}(\mathbf{u})\right) .
$$

Thus the key is to find the coefficients $c_{k}$ in (5.8); the first one is determined by the cusp shapes, which are both square, and hence $c_{1}=i$.

Using Gröbner bases [8] to eliminate variables from (5.6) and (5.7), we find the following relationship between $V_{1}, U_{1}$ and $U_{2}$ :

(5.10) $U_{2} V_{1}^{2}+U_{1}^{6} U_{2} V_{1}^{4}-2 U_{1} U_{2} V_{1}^{2}\left(3-V_{1}+V_{1}^{2}\right)-2 U_{1}^{5} U_{2} V_{1}^{2}\left(1-V_{1}+3 V_{1}^{2}\right)$

$$
-U_{1}^{4}\left(\left(-1+V_{1}\right)^{4} V_{1}+U_{2}^{2}\left(-1+V_{1}\right)^{4} V_{1}+U_{2}\left(-1+4 V_{1}-15 V_{1}^{2}+12 V_{1}^{3}-15 V_{1}^{4}\right)\right)
$$$$
+U_{1}^{2} V_{1}\left(-\left(-1+V_{1}\right)^{4}-U_{2}^{2}\left(-1+V_{1}\right)^{4}+U_{2} V_{1}\left(15-12 V_{1}+15 V_{1}^{2}-4 V_{1}^{3}+V_{1}^{4}\right)\right)
$$$$
+2 U_{1}^{3} V_{1}\left(\left(-1+V_{1}\right)^{4}+U_{2}^{2}\left(-1+V_{1}\right)^{4}-U_{2}\left(1+4 V_{1}+4 V_{1}^{3}+V_{1}^{4}\right)\right)=0
$$

If we substitute $U_{k}=e^{u_{k}}=1+u_{k}+u_{k}^{2} / 2+u_{k}^{3} / 6+O\left(|\mathbf{u}|^{4}\right)$ and

$$
\begin{aligned}
V_{1}=e^{v_{1}} & =\exp \left(i u_{1}+2 c_{2} u_{1}^{3}+c_{3} u_{1} u_{2}^{2}+O\left(|\mathbf{u}|^{4}\right)\right) \\
& =1+i u_{1}-\frac{1}{2} u_{1}^{2}+\frac{12 c_{2}-i}{6} u_{1}^{3}+c_{3} u_{1} u_{2}^{2}+O\left(|\mathbf{u}|^{4}\right)
\end{aligned}
$$

into (5.10), we get

$$
-\left(1+(8-8 i) c_{3}\right) u_{1}^{6} u_{2}^{2}-\left(\frac{1+2 i}{3}+(16-16 i) c_{2}\right) u_{1}^{8}+O\left(|\mathbf{u}|^{9}\right)=0
$$

which forces $c_{2}=(-3+i) / 96$, and $c_{3}=-(1+i) / 16$. In particular,

$$
v_{1}=i u_{1}+\frac{-3+i}{48} u_{1}^{3}-\frac{1+i}{16} u_{1} u_{2}^{2}+O\left(|\mathbf{u}|^{4}\right) \text {. }
$$


Now (5.9) becomes

(5.11) $\operatorname{Vol}\left(W_{\mathbf{u}}\right)=\operatorname{Vol}(W)-\frac{1}{4}\left(u_{1} \bar{u}_{1}+u_{2} \bar{u}_{2}\right)-\frac{1}{64} \operatorname{Im}\left(\frac{3+i}{3}\left(u_{1} \bar{u}_{1}^{3}+u_{2} \bar{u}_{2}^{3}\right)\right.$

$$
\begin{array}{r}
\left.+(1-i)\left(u_{1} \bar{u}_{1} \bar{u}_{2}^{2}+\bar{u}_{1}^{2} u_{2} \bar{u}_{2}\right)-\left(\frac{3-i}{6}\left(u_{1}^{4}+u_{2}^{4}\right)+(1+i) u_{1}^{2} u_{2}^{2}\right)\right) \\
+O\left(|\mathbf{u}|^{6}\right)
\end{array}
$$

We can now use this formula to estimate $\operatorname{Vol}\left(W_{\phi}\right)$ as follows; here, we do the case $\epsilon(\phi)=0$, and leave the other to the reader. By Lemma 2.10, $W_{\phi}$ is obtained by filling along

$$
\gamma_{1}=(a-2 b) \mu_{1}+(2 a+b) \lambda_{1} \quad \text { and } \quad \gamma_{2}=(-2 a+b) \mu_{2}+(a+2 b) \lambda_{2} .
$$

Thus if $\mathbf{u}$ is the parameter realizing the hyperbolic structure on $W_{\phi}$ we have, remembering that $v_{k}=\log \left(h\left(-\lambda_{k}\right)\right)$, the following relationship

(5.12) $(a-2 b) u_{1}-(2 a+b) v_{1}=2 \pi i \quad$ and $\quad(-2 a+b) u_{2}-(a+2 b) v_{2}=2 \pi i$.

Following [19, Section 6], we introduce quantities

$$
A_{1}=(a-2 b)-(2 a+b) i \quad \text { and } \quad A_{2}=(-2 a+b)-(a+2 b) i
$$

and use (5.12) to find that

$$
\begin{aligned}
& u_{1}=\frac{2 \pi i}{A_{1}}-\frac{\pi^{3}(2 a+b) i}{2 A_{1}^{2}}\left(\frac{1-i / 3}{A_{1}^{2}}+\frac{1+i}{A_{2}^{2}}\right)+O\left(\frac{1}{a^{5}}\right) \\
& u_{2}=\frac{2 \pi i}{A_{2}}-\frac{\pi^{3}(a+2 b) i}{2 A_{2}^{2}}\left(\frac{1+i}{A_{1}^{2}}+\frac{1-i / 3}{A_{2}^{2}}\right)+O\left(\frac{1}{a^{5}}\right) .
\end{aligned}
$$

Substituting this into (5.11) and expanding gives

$$
\operatorname{Vol}\left(W_{\phi}\right)=\operatorname{Vol}(W)-\frac{2 \pi^{2}}{5\left(a^{2}+b^{2}\right)}-\frac{\pi^{4}\left(7 a^{4}+144 a^{2} b^{2}+7 b^{4}\right)}{1875\left(a^{2}+b^{2}\right)^{4}}+O\left(\frac{1}{a^{6}}\right)
$$

as claimed.

We now use this last result to prove the following, which immediately gives Theorem 5.1 when combined with Theorem 5.4 and Definition 3.3.

5.14 Theorem For all large $g$ the following is true. Suppose $\phi=a x^{*}+b_{1} y^{*}$ and $\psi=a x^{*}+b_{2} y^{*}$ are both in $\mathcal{A}_{g}$ or both in $\mathcal{E}_{g}$. If $b_{1}>b_{2}$ then $\operatorname{Vol}\left(W_{\phi}\right)>\operatorname{Vol}\left(W_{\psi}\right)$. 
Proof First suppose $\phi$ and $\psi$ are in $\mathcal{A}_{g}$. By the part of Theorem 5.5 that followed directly from Lemma 5.2, we have

$\operatorname{Vol}\left(W_{\phi}\right)-\operatorname{Vol}\left(W_{\psi}\right)=\frac{2 \pi^{2}}{5} \frac{b_{1}^{2}-b_{2}^{2}}{\left(a^{2}+b_{1}^{2}\right)\left(a^{2}+b_{2}^{2}\right)}+O\left(\frac{1}{a^{4}}\right) \geq \frac{\pi^{2}}{10} \frac{b_{1}^{2}-b_{2}^{2}}{a^{4}}+O\left(\frac{1}{a^{4}}\right)$.

Suppose the error term above is $<C / a^{4}$. As $b_{1}>b_{2}$ are integers, $b_{1}^{2}-b_{2}^{2}>2 b_{2}$, so if $b_{2}>10 C$ then $\operatorname{Vol}\left(W_{\phi}\right)>\operatorname{Vol}\left(W_{\psi}\right)$. Thus we can assume that $b_{1}$ and $b_{2}$ are uniformly bounded, independent of $a$.

Now the more refined part of Theorem 5.5 gives that

$\operatorname{Vol}\left(W_{\phi}\right)-\operatorname{Vol}\left(W_{\psi}\right)=\frac{2 \pi^{2}}{5} \frac{b_{1}^{2}-b_{2}^{2}}{\left(a^{2}+b_{1}^{2}\right)\left(a^{2}+b_{2}^{2}\right)}+\frac{\pi^{4} P\left(a, b_{1}, b_{2}\right)}{\left(a^{2}+b_{1}^{2}\right)^{4}\left(a^{2}+b_{2}^{2}\right)^{4}}+O\left(\frac{1}{a^{6}}\right)$

where $P \in \mathbb{Z}\left[a, b_{1}, b_{2}\right]$ has degree 10 in $a$. Since the $b_{i}$ are uniformly bounded, this gives

$$
\operatorname{Vol}\left(W_{\phi}\right)-\operatorname{Vol}\left(W_{\psi}\right)=\frac{2 \pi^{2}}{5} \frac{b_{1}^{2}-b_{2}^{2}}{\left(a^{2}+b_{1}^{2}\right)\left(a^{2}+b_{2}^{2}\right)}+O\left(\frac{1}{a^{6}}\right) \geq \frac{\pi^{2}}{10 a^{4}}+O\left(\frac{1}{a^{6}}\right)
$$

and hence $\operatorname{Vol}\left(W_{\phi}\right)>\operatorname{Vol}\left(W_{\psi}\right)$ for large $g$.

If instead $\phi$ and $\psi$ are in $\mathcal{E}_{g}$, the only significant difference is that $P$ has degree 11 in $a$ and thus one gets, when the $b_{i}$ are bounded, that

$$
\operatorname{Vol}\left(W_{\phi}\right)-\operatorname{Vol}\left(W_{\psi}\right)=\frac{26 \pi^{2}}{5} \frac{b_{1}^{2}-b_{2}^{2}}{\left(a^{2}+b_{1}^{2}\right)\left(a^{2}+b_{2}^{2}\right)}+O\left(\frac{1}{a^{5}}\right)
$$

which still suffices to prove the theorem.

\section{The monodromy of $W_{\phi}$}

In this section, we compute the Teichmüller polynomial for a face of the Thurston norm ball of $W$, and use this to calculate the dilatation for each $M_{g}$, as well as the other closed 3-manifolds fibering over the circle which are obtained by filling $W$. For background and definitions, see McMullen's original paper [17]. Specifically, in the notation of Section 2, we have:

6.1 Theorem Consider $\phi=a x^{*}+b y^{*} \in H^{1}(W ; \mathbb{Z})$ with $0 \leq b<a$ and $\operatorname{gcd}(a, b)=1$. If $(a, b) \notin\{(1,0),(2,1),(3,1),(4,3)\}$, then $W_{\phi}$ is a hyperbolic 3 -manifold which is a $\Sigma_{g}$-bundle, where $g=a-2 \epsilon(\phi)$. The invariant foliations for the monodromy have 
$5^{\epsilon_{k}(\phi)}$ singularities with $5^{-\epsilon_{k}(\phi)}\left(2 a+(-1)^{k+1} b\right)$ prongs for $k=1,2$. The dilatation of the monodromy is the largest real root of

$$
\Lambda_{a, b}(t)=t^{2 a}-t^{a+b}-t^{a}-t^{a-b}+1 .
$$

Finally, the invariant foliations are (co)orientable exactly when $a$ is odd and $b$ is even.

Combining this with Proposition 3.4 immediately gives:

6.2 Corollary The dilatation $\lambda_{g}$ of the monodromy of $M_{g}$ is the largest real root of $a_{g}(t)$ where $a_{g}(t)$ is given by the following:

(1) If $g \equiv 0,1 \bmod 5$, then $a_{g}(t)=\Lambda_{g+2,1}(t)=t^{2 g+4}-t^{g+3}-t^{g+2}-t^{g+1}+1$.

(2) If $g \equiv 3 \bmod 5$, then

(a) $a_{g}(t)=\Lambda_{g, 2}=t^{2 g}-t^{g+2}-t^{g}-t^{g-2}+1$ when $g \equiv 3,13,23 \bmod 30$.

(b) $a_{g}(t)=\Lambda_{g, 3}=t^{2 g}-t^{g+3}-t^{g}-t^{g-3}+1$ when $g \equiv 8,28 \bmod 30$.

(c) $a_{g}(t)=\Lambda_{g, 5}=t^{2 g}-t^{g+5}-t^{g}-t^{g-5}+1$ when $g \equiv 18 \bmod 30$.

(3) If $g \equiv 2,4 \bmod 5$, then $a_{g}(t)=\Lambda_{g+2, b}=t^{2 g+4}-t^{g+b+2}-t^{g+2}-t^{g-b+2}+1$, where $b$ is chosen to be the smallest number coprime to $g+2$ satisfying $b \equiv$ $2,3 \bmod 5$.

\subsection{The Teichmüller polynomial}

Our starting point for Theorem 6.1 is to view $W$ as a mapping torus of the 5-punctured disk $D$ with monodromy $\psi=\sigma_{1}^{3} \sigma_{2} \sigma_{3} \sigma_{4}$ (see Figure 3). Using the Bestvina-Handel algorithm, as implemented by Hall [9], we found the invariant train track $\tau$ shown in Figure 4.

The monodromy $\psi$ permutes the vertices of $\tau$ via the 5 -cycle $(1,3,5,4,2)$, and just permutes the vertex loops in the compatible way. On the other edges, $\psi$ wraps them as follows, where "." denotes concatenation of paths from left to righthand (as with the usual conventions for fundamental group):

$$
e_{6} \mapsto e_{9} \cdot e_{5} \cdot e_{8}^{-1} \quad e_{7} \mapsto e_{6} \cdot e_{4}^{-1} \cdot e_{7}^{-1} \quad e_{8} \mapsto e_{6} \quad e_{9} \mapsto e_{8}^{-1} \cdot e_{2}^{-1} \cdot e_{7}
$$

Following [17], we can compute the Teichmüller polynomial by considering the lift of $\psi$ to a certain abelian cover $\widetilde{D} \rightarrow D$, and then considering its action on the space of weights of $\tilde{\tau}$. This is analogous to computing the Burau representation or the Alexander polynomial, and what follows can be thought of in terms of Fox calculus. Here, $\widetilde{D}$ comes from the map from $\pi_{1}(D)$ to the infinite cyclic group $\langle t\rangle$ which maps 


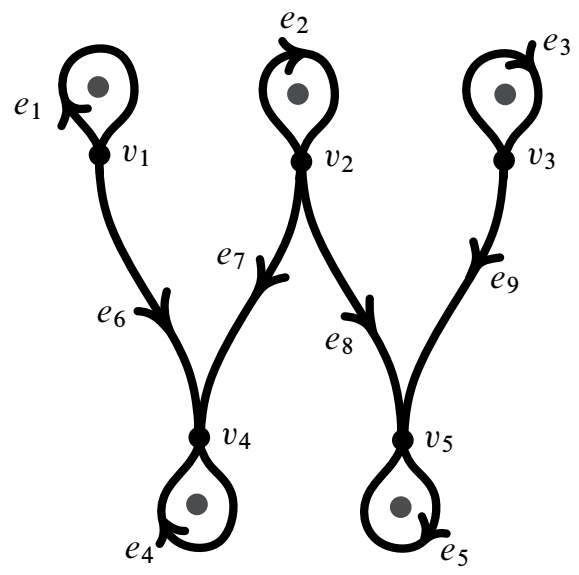

Figure 4: The invariant train track for $\psi=\sigma_{1}^{3} \sigma_{2} \sigma_{3} \sigma_{4}$

a clockwise loop about a single puncture to $t$. Observing how the $t$-grading shifts along the image edges in (6.4), we find that in $\widetilde{D}$ :

$$
\tilde{v}_{1} \mapsto \widetilde{v}_{3} \quad \widetilde{v}_{2} \mapsto t^{2} \widetilde{v}_{1} \quad \widetilde{v}_{3} \mapsto t^{3} \widetilde{v}_{5} \quad \widetilde{v}_{4} \mapsto t \widetilde{v}_{2} \quad \widetilde{v}_{5} \mapsto t^{2} \widetilde{v}_{4}
$$

The action of $\tilde{\psi}$ on the first five edges is determined by the vertex action, and the others are

$$
\begin{array}{lll}
\tilde{e}_{6} \mapsto t \widetilde{e}_{5}+t \widetilde{e}_{8}+\widetilde{e}_{9} & \widetilde{e}_{7} \mapsto t^{2}\left(\widetilde{e}_{4}+\widetilde{e}_{6}+t^{-1} \widetilde{e}_{7}\right) \\
\widetilde{e}_{8} \mapsto t^{2} \widetilde{e}_{6} & \widetilde{e}_{9} \mapsto t^{3}\left(\widetilde{e}_{2}+t^{-1} \widetilde{e}_{7}+\widetilde{e}_{8}\right)
\end{array}
$$

Now by [17], to get the Teichmüller polynomial we simply compute

$$
\Theta(u, t)=\frac{\operatorname{det}\left(u I-P_{E}(t)\right)}{\operatorname{det}\left(u I-P_{V}(t)\right)}=-u t^{5}+t^{6}-u^{2} t^{3}+u^{4}-u^{3} t,
$$

where $u$ is a certain element of $H_{1}(W)$ which goes once around the bundle. To put this in our usual basis for $H_{1}(W)$, first note from Figure 3 that $t=x-2 y$ and the element of $H^{1}(W ; \mathbb{Z})$ corresponding to this fibration is $\phi=2 x^{*}+y^{*}$. Since $\phi(u)=1$ and $\Theta$ determines the righthand face of the Thurston norm ball given in Figure 2(c), it follows that $u=2 x-3 y$ and

$$
\Theta(x, y)=x+x^{-1}-1-y-y^{-1}=-\Delta_{W}(-x, y) .
$$

\subsection{Invariant foliations}

Consider $\phi=a x^{*}+b y^{*} \in H^{1}(W ; \mathbb{Z})$ over the interior of the righthand fibered face $F$, ie $a>0$ and $|b|<a$. Consider the monodromy $\psi: \Sigma \rightarrow \Sigma$ of the corresponding 
fibration of $W$ (here $\Sigma$ is a certain surface with boundary). We wish to understand the topology of the invariant foliations of $\psi$ so that we can understand the bundle structure of the Dehn-filled manifold $W_{\phi}$, and use (6.5) to compute the stretch factor.

For concreteness, let us focus on the stable foliation $\mathcal{F} \subset \Sigma$. This foliation can be suspended via $\psi$ to a 2 -dimensional foliation $\tilde{\mathcal{F}}$ of $W$ with a finite number of singular leaves. Fried showed that $\tilde{\mathcal{F}}$ only depends, up to isotopy, on the fibered face and not the particular choice of $\phi$ [6]. Thus from the single case shown in Figure 4, we see that the singularities of $\widetilde{\mathcal{F}}$ occur only at the boundary of $W$. Moreover, there is only one singular leaf touching $\partial_{1} W$. As noted in Section 2, there is an involution of $W$ corresponding to $x \mapsto x, y \mapsto Y$. This preserves $F$ hence and $\tilde{\mathcal{F}}$. Because of (2.5), the involution interchanges the cusps via $\left(\mu_{1} \leftrightarrow \lambda_{2}, \lambda_{1} \leftrightarrow-\mu_{2}\right)$. Thus $\tilde{\mathcal{F}}$ also has only one singular leaf touching $\partial_{2} W$.

Let $\delta_{k}$ be the curve in $\partial_{k} W$ corresponding to the boundary of the singular leaf (in the lamination context, $\delta_{k}$ is called the degeneracy slope of $\tilde{\mathcal{F}}$ ). Because of the involution, we have

$$
\delta_{1}=c \mu_{1}+d \lambda_{1} \quad \text { and } \quad \delta_{2}=-d \mu_{2}+c \lambda_{2} \quad \text { for some } c, d \in \mathbb{Z} .
$$

Returning to the foliation $\mathcal{F}$ in the fiber surface $\Sigma$, we now know it has no singularities in the interior. Moreover, if $\gamma_{k}$ is a boundary component of the fiber surface $\Sigma$ lying on $\partial_{k} W$, the number of singular prongs is just the intersection number $\left|\gamma_{k} \cdot \delta_{k}\right|$. From the case of $\phi=2 x^{*}+1$ above, using Lemma 2.10 it easily follows that $\delta_{1}=\mu_{1}$ and $\delta_{2}=\lambda_{2}$.

For a general $\phi=a x^{*}+b y^{*}$ over $F$ with $b \geq 0$, by (2.9) and Lemma 2.10, the fiber surface has $5^{\epsilon_{k}(\phi)}$ boundary components along $\partial_{k} W$ and the foliation $\mathcal{F}$ has singularities with $5^{-\epsilon_{k}(\phi)}\left(2 a+(-1)^{k+1} b\right)$ prongs. We are now ready to prove Theorem 6.1 .

Proof of Theorem 6.1 Fix $\phi=a x^{*}+b y^{*}$ with $0 \leq b<a$ and $\operatorname{gcd}(a, b)=1$. By Theorem 2.8, the filled manifold $W_{\phi}$ fibers over the circle in a unique way, and the fiber genus is $a-2 \epsilon(\phi)$. An easy check shows that if $(a, b) \notin\{(1,0),(2,1),(3,1)\}$, then the genus is at least 2. Similarly, it is straightforward from the above formulae to see that for $(a, b) \notin\{(2,1),(3,1),(4,3)\}$, there are always at least two prongs at each boundary component. Thus when we Dehn fill to get $W_{\phi}$, the monodromy $\psi$ remains pseudo-Anosov with the same invariant foliations and dilatation factor. By [17], that dilatation $\lambda$ is the largest real root of

$$
\Theta\left(x=t^{\phi(x)}, y=t^{\phi(y)}\right)=t^{a}+t^{-a}-1-t^{b}-t^{-b}
$$

or, multiplying through by $t^{a}$, of $\Lambda(t)=t^{2 a}-t^{a+b}-t^{a}-t^{a-b}+1$. 
Next, we need to check when the foliations are orientable. Suppose $b$ is odd. Since only one of $\epsilon_{k}(\phi) \neq 0$, it follows from the above formulae that there are singularities with either $2 a+b$ or $2 a-b$ prongs, and both of these numbers are odd. Thus the foliation is nonorientable. If $a$ is even, then $b$ is odd and so the foliation is again nonorientable. In the remaining case where $a$ is odd and $b$ is even, one has by (2.3) that the Alexander polynomial of $W_{\phi}$ is related to the polynomial $\Lambda(t)$ defining $\lambda$ by

$$
\Delta_{W_{\phi}}=\Delta_{W}\left(x=t^{a}, y=t^{b}\right)=t^{2 a}+t^{a+b}+t^{a}+t^{a-b}+1=\Lambda(-t) .
$$

Thus in this case, the spectral radius of the action of $\psi$ on the homology of the fiber is the same as the dilatation $\lambda$. Thus the foliation must be orientable (see eg [16, page 5]). Summarizing, for $\phi \notin\{(1,0),(2,1),(3,1),(4,3)\}$, the manifold $W_{\phi}$ fibers over the circle with fiber a closed surface of genus at least 2 and pseudo-Anosov monodromy having the claimed data. By Thurston, the bundle $W_{\phi}$ is thus hyperbolic, completing the proof $[22 ; 20]$.

\section{Minimizing dilatation}

Having shown earlier which surgeries on $W$ minimize volume, we now turn to determining which minimize the dilatation of the monodromy, and find:

7.1 Theorem For large $g$, the monodromy of $M_{g}$ minimizes dilatation among all $\Sigma_{g}$-bundles obtained from filling $W$.

Moreover, the asymptotic behavior of these minimal dilatations is:

7.2 Theorem Let $\lambda_{g}$ denote the dilatation of $M_{g}$. Then $\lim _{g \rightarrow \infty} \lambda_{g}^{g}=(3+\sqrt{5}) / 2=$ $1+\gamma$, where $\gamma$ is the golden ratio.

To understand how the dilatation depends on $\phi \in H^{1}(W ; \mathbb{Z})$, we follow [17, Section 5]. In particular, for such $\phi$ lying over the rightmost face of the Thurston norm ball, let $K(\phi)$ be the dilatation of the monodromy of the corresponding bundle. If we set $Z(\phi)=1 / \log (K(\phi)$, then this extends to a nonnegative continuous function defined on $\left\{a x^{*}+b y^{*} \in H^{1}(W ; \mathbb{R}) \mid a \geq 0\right.$ and $\left.|b| \leq a\right\}$. The function $Z$ is homogeneous with $Z(c \phi)=c Z(\phi)$ and is strictly concave. It vanishes exactly along the rays $b= \pm a$ and is real-analytic away from them. Thus $Z$ is completely determined by $z:[-1,1] \rightarrow \mathbb{R}$ given by $z(t)=Z\left(x^{*}+t y^{*}\right)$. As noted in Section 2, there is an involution of $W$ which acts on $H^{1}(W ; \mathbb{R})$ by flipping across the $x^{*}$-axis. This involution preserves $K$ and hence $Z$; it follows that $z$ is even and, since it is strictly concave, has a unique maximum at 0 . 
Proof of Theorem 7.1 Fix a genus $g$, and recall from Section 3 that the $\phi=a x^{*}+b y^{*}$ giving $\Sigma_{g}$-bundles fall into two classes, namely $\mathcal{A}_{g}$ where $a=g$ and $\mathcal{E}_{g}$ where $a=g+2$. We seek to minimize $K(\phi)$, or correspondingly maximize $Z(\phi)$. From the discussion above, among $\phi$ within just one of $\mathcal{A}_{g}$ or $\mathcal{E}_{g}$, it is clear that $Z(\phi)$ is maximized when $b$ is least.

If $g \equiv 3 \bmod 5$, then $\mathcal{E}_{g}$ is empty and $M_{g}$ comes from the $\phi \in \mathcal{A}_{g}$ with least $b$. Thus $Z(\phi)$ is maximized for $M_{g}$ and we're done with this case.

Otherwise, let $\phi_{g}=(g+2) x^{*}+b y^{*}$ denote the element of $\mathcal{E}_{g}$ with smallest $b$ so that $M_{g}=W_{\phi_{g}}$. We will show that for large $g$ one has

$$
Z\left(g x^{*}\right)<Z\left(\phi_{g}\right) .
$$

This suffices since we know $Z(\phi)<Z\left(g x^{*}\right)$ for any $\phi \in \mathcal{A}_{g}$. By the homogeneity of $Z$, the claim (7.3) is equivalent to

$$
g z(0)<(g+2) z\left(\frac{b}{g+2}\right) \text { or } \frac{g}{g+2}<\frac{z(b /(g+2))}{z(0)} .
$$

Now $z$ is real-analytic and $z^{\prime}(0)=0$ as it has a max at 0 . Expanding $z$ in a power series about 0 , and applying the bound on $b$ from Proposition 3.4(5), we get for large $g$ that

$$
\frac{z(b /(g+2))}{z(0)}>1-C_{1}\left(\frac{b}{(g+2)}\right)^{2}>1-C_{2} \frac{\log ^{2} g}{(g+2)^{2}} .
$$

Comparing this with $g /(g+2)=1-2 / g+O\left(g^{-2}\right)$ gives the claim (7.4) for large $g$, proving the theorem.

Proof of Theorem 7.2 Let $\phi_{g}$ be the class giving $M_{g}$. The properties of $Z$ mentioned above mean that $K$ also extends to a continuous function with $K(c \phi)=K(\phi)^{1 / c}$. Thus

$$
\lambda_{g}^{g}=K\left(\phi_{g}\right)^{g}=K\left((1 / g) \phi_{g}\right) .
$$

By Proposition 3.4(5), we know that if $\phi_{g}=a x^{*}+b y^{*}$ then $b$ is $O(\log g)$. Thus we see that $(1 / g) \phi_{g}$ converges to $x^{*}$ as $g \rightarrow \infty$. Hence by continuity $\lim _{g \rightarrow \infty} \lambda_{g}^{g}=K\left(x^{*}\right)$. The latter is the largest root of

$$
\Theta(x=t, y=1)=t^{2}-3 t+1
$$

which is $(3+\sqrt{5}) / 2=1+\gamma$ as claimed.

We now turn to our partial answer to Question 1.6. 
7.5 Theorem Assume Conjecture 4.1. Let $\delta_{g}$ be the smallest dilatation of any pseudo-Anosov of $\Sigma_{g}$. Then for all large $g \equiv 3 \bmod 5$, no pseudo-Anosov $\psi_{g}$ with dilatation $\delta_{g}$ gives a hyperbolic $\Sigma_{g}$-bundle of least volume.

Proof Assuming Conjecture 4.1 gives by Theorem 1.2 that we know there is a unique $\Sigma_{g}$-bundle of least volume when $g$ is large, namely $M_{g}$. Let $\lambda_{g}$ be the corresponding dilatation. We compare this to the pseudo-Anosov of $\Sigma_{g}$ constructed by Hironaka in [12, Theorem 1.4], whose dilatation we denote by $\epsilon_{g}$. In particular, we just need to show that $\epsilon_{g}<\lambda_{g}$.

Following the notation of [12], let $\lambda_{(b, a)}$ be the largest root of the polynomial in Theorem 6.1. Then the dilatation $\epsilon_{g}$ is either $\lambda_{(1, g+1)}$ or $\lambda_{(3, g+1)}$. As $g \equiv 3 \bmod 5$, Corollary 6.2 gives that $\lambda_{g}=\lambda_{(b, g)}$ where $b \in\{2,3,5\}$. By Propositions 4.1 and 4.2 of [12], one has

$$
\epsilon_{g} \leq \lambda_{(3, g+1)} \leq \lambda_{(1, g)}<\lambda_{g}
$$

as needed.

Finally, we identify the minimal dilatation $\delta_{g}^{+}$of any pseudo-Anosov of $\Sigma_{7}$ with orientable invariant foliations.

Proof of Theorem 1.11 Consider the monodromy $\psi$ of the bundle $M_{7}$, which corresponds to $\phi=9 x^{*}+2 y^{*}$. By Theorem 6.1, the invariant foliations of $\psi$ are orientable, and the dilatation $\lambda_{7} \approx 1.11548110945659$ is the largest root of

$$
\begin{aligned}
& t^{18}-t^{11}-t^{9}-t^{7}+1=\left(t^{4}-t^{3}+t^{2}-t+1\right) \\
& \cdot\left(t^{14}+t^{13}-t^{9}-t^{8}-t^{7}-t^{6}-t^{5}+t+1\right) .
\end{aligned}
$$

Now $\lambda_{7}$ is a root of the second irreducible factor above, which is exactly the polynomial used in [16] to give a lower bound on $\delta_{7}^{+}$. Thus $\delta_{7}^{+}=\lambda_{7}$, as claimed.

\section{References}

[1] I Agol, The minimal volume orientable hyperbolic 2-cusped 3-manifolds, Proc. Amer. Math. Soc. 138 (2010) 3723-3732

[2] P J Callahan, MV Hildebrand, J R Weeks, A census of cusped hyperbolic 3manifolds, Math. Comp. 68 (1999) 321-332 MR1620219

[3] J-H Cho, J-Y Ham, The minimal dilatation of a genus-two surface, Experiment. Math. 17 (2008) 257-267 MR2455699

[4] M Culler, N M Dunfield, J R Weeks, SnapPy, a computer program for studying the geometry and topology of 3-manifolds Available at http://snappy.computop.org 
[5] B Farb, C Leininger, D Margalit, Small dilatation pseudo-Anosovs and 3-manifolds arXiv:0905.0219

[6] D Fried, Fibrations over $S^{1}$ with pseudo-Anosov monodromy, from: "Travaux de Thurston sur les surfaces", Séminaire Orsay, Astérisque 66, Soc. Math. France (1979) 251-266 MR568308

[7] D Gabai, R Meyerhoff, P Milley, Mom technology and hyperbolic 3-manifolds, from: "In the tradition of Ahlfors-Bers. V", (M Bonk, J Gilman, H Masur, Y Minsky, M Wolf, editors), Contemp. Math. 510, Amer. Math. Soc. (2010) 84-107 MR2581832

[8] D R Grayson, M E Stillman, Macaulay 2, a software system for research in algebraic geometry Available at http://www.math.uiuc.edu/Macaulay2/

[9] T Hall, trains3, an implementation of Bestvina and Handel's algorithm Available at http://www.liv.ac.uk/maths/PURE/MIN_SET/CONTENT/members/T_Hall

[10] T Hall, S Schleimer, Hyperbolic genus two bundles with monodromy of length ten or less (2002) Available at http://www.warwick.ac.uk/ masgar/Maths/ twister.html

[11] D Heard, E Pervova, C Petronio, The 191 orientable octahedral manifolds, Experiment. Math. 17 (2008) 473-486 MR2484431

[12] E Hironaka, Small dilatation pseudo-Anosov mapping classes coming from the simplest hyperbolic braid, to appear in Algebr. Geom. Topol. arXiv:0909.4517

[13] A A Karatsuba, Basic analytic number theory, Springer, Berlin (1993) MR1215269 Translated from the second (1983) Russian edition and with a preface by M B Nathanson

[14] E Kin, S Kojima, M Takasawa, Entropy versus volume for pseudo-Anosovs, Experiment. Math. 18 (2009) 397-407 MR2583541

[15] E Kin, M Takasawa, Pseudo-Anosovs on closed surfaces having small entropy and the Whitehead sister link exterior arXiv:1003.0545

[16] E Lanneau, J-L Thiffeault, On the minimum dilatation of pseudo-Anosov homeomorphisms on surfaces of small genus, to appear in Ann. Inst. Fourier (Grenoble) arXiv: 0905.1302

[17] C T McMullen, Polynomial invariants for fibered 3-manifolds and Teichmüller geodesics for foliations, Ann. Sci. École Norm. Sup. (4) 33 (2000) 519-560 MR1832823

[18] C T McMullen, The Alexander polynomial of a 3-manifold and the Thurston norm on cohomology, Ann. Sci. École Norm. Sup. (4) 35 (2002) 153-171 MR1914929

[19] W D Neumann, D Zagier, Volumes of hyperbolic three-manifolds, Topology 24 (1985) 307-332 MR815482

[20] J-P Otal, Le théorème d'hyperbolisation pour les variétés fibrées de dimension 3, Astérisque 235 (1996) MR1402300 
[21] R C Penner, Bounds on least dilatations, Proc. Amer. Math. Soc. 113 (1991) 443-450 MR1068128

[22] W P Thurston, Hyperbolic structures on 3-manifolds, II: Surface groups and 3manifolds which fiber over the circle arXiv:math.GT/9801045

[23] W P Thurston, A norm for the homology of 3-manifolds, Mem. Amer. Math. Soc. 59 (1986) i-vi and 99-130 MR823443

[24] R W Venzke, Braid forcing, hyperbolic geometry, and pseudo-Anosov sequences of low entropy, PhD thesis, Caltech (2008) Available at http://resolver.caltech.edu/ CaltechETD : etd-05292008-085545

[25] J R Weeks, SnapPea: A computer program for creating and studying hyperbolic 3manifolds Available at http://www.geometrygames.org/SnapPea/

Department of Mathematics MC-382, University of Illinois

1409 W Green Street, Urbana, IL 61801, USA

tophat1234@hotmail.com, nathan@dunfield.info

http://dunfield.info

Received: 10 September 2010 